\title{
Approach for Modelling Process Effects during Friction Stir Welding of Composite Extruded Profiles
}

\author{
Michael F. Zaeh ${ }^{1, a}$ and Alexander Schober ${ }^{1, b}$ \\ ${ }^{1}$ Institute for Machine Tools and Industrial Management (iwb) \\ Technische Universität München, Boltzmannstr. 15, D-85748 Garching, Germany \\ amichael.zaeh@iwb.tum.de, ${ }^{\mathrm{b}}$ alexander.schober@iwb.tum.de
}

\begin{abstract}
Keywords: Finite element analysis, friction stir welding, composite aluminium extrusions, light weight construction
\end{abstract}

\begin{abstract}
In the field of light weight frame structures the assurance of dimensional accuracy and the prediction of structural properties especially during and after welding processes are of great importance. The problem in this regard mostly arises from the used welding technique which is characterised by complex interactions of various parameters. A simulative approach is useful in order to predict the structural behaviour and to improve the geometrical quality of joined light weight components after welding. As such, it contributes to reduce process adjustments in the early stage of the product life cycle, and therefore helps to save time and costs. In this paper an approach for modelling the innovative joining process of composite extruded profiles by friction stir welding is presented.
\end{abstract}

\section{Introduction}

This paper discusses some results that were achieved in sub-project B4 within the Transregional Collaborative Research Centre 10. The scientific objective of this project is the simulation of the interactions between the considered thermal joining processes (friction stir welding and laser beam welding) and the component structure [1]. In particular the joining of composite extruded profiles will be investigated. In doing so, various new aspects have to be considered. For instance, the different material parameters - in particular the coefficient of thermal expansion - of the matrix and the reinforcing elements cause the generation of stresses during a heat treatment, particularly when a welding process is performed. In this regard it is of interest to study low heat joining technologies such as friction stir welding. The benefit of applying such an innovative process should be proved. Processes during which a great amount of heat is applied to the treated components will be examined as well, in order to test the limits of their applicability. The particular joining technique that will be studied in this regard is laser beam welding. The evaluation of the applicability of these joining techniques for various profile types will be clarified in cooperation with other sub-projects that investigate the process sensibility, particularly sub-project A11. It is intended to compare experimental results to those of appropriate simulations for mutual benefit, meaning, on the one hand, to gain information for optimising the process and, on the other hand, to validate the results obtained from the simulation. Depending on the geometrical properties and the used materials of the various profiles, methods will be derived to calculate the state of the regarded component during and after the examined welding process. 


\section{Presentation of the considered example}

The joining process of two composite extruded profiles by means of friction stir welding provides an application example for the examination discussed within this paper. This example describes the experiments that were conducted by sub-project A11, which studies the joining techniques of friction stir welding and laser beam welding. In the course of that sub-project various experiments were and will be conducted at the authors' institute. As a starting point the setup of the above mentioned experiment provides the basis for modelling. In detail this experiment involves a butt joint of two extruded composite sheet metals - each made of an aluminium matrix reinforced with six steel wires that are distributed equidistantly - with the dimensions $35 \times 56 \times 5 \mathrm{~mm}$. These extruded composite profiles are shown in Fig. 1. A detailed description of the experimental setup is discussed in a following paragraph. A transient temperature field is calculated in a thermophysical simulation of the welding process until cooling of the structure to the ambient temperature. Within a thermomechanical simulation, the welding distortion and the residual stresses of the workpiece are finally calculated considering the influence of the reinforcing elements in the structure and the clamping conditions during friction stir welding.

\section{Setup of the simulation}

To conduct the above mentioned computations the finite element method is applied. Therefore the complex physical effects that occur during a welding operation become computable due to a discretisation in space and time - by using a finite element mesh and a partition in discrete time steps respectively. The increasing available computing power and the advancement of commercial software both contribute to increase the accuracy of the results of a simulation and to decrease the amount of time to compute them. From another point of view, it is possible to examine structures with more complex geometries or include more physical effects by coupling different computations.

To compute the structural results, commercial software is used. There are two main computation steps in this regard. Firstly the transient temperature field is computed. The definition of the intensity of the heat source as an input parameter is determined iteratively. To validate the results of the simulation various ways are possible. These include examinations of cross sections of the weld seam, temperature measurements during the welding process, evaluations of experience based data sets or a combination of the above. Secondly the effects of the thermomechanical and mechanical loads during the joining process within the component structure are calculated. The result herefrom is a global state of residual stresses, which combines the consequences of the various influences. In the simulation it is important to take into consideration both the modelling of the clamping situation and the stress formation that occurs on the interface between the different materials of matrix and reinforcing elements.

All simulations are conducted using commercial software. As a starting point all meshing tasks are handled with HyperMesh (Altair) while all further modelling tasks and computations are performed with SYSWELD (ESI Group). Further software tools will be used as well in the course of the project. Therefore, it will be possible to compare results even between two or more numerical simulations using different software tools. In addition numerous experiments will be conducted to validate the numerical results. 


\section{Approach for modelling}

The basic setup of the described friction stir welding experiment - size of the sheets to be joined, clamping conditions etc. - will be adopted for the modelling. Furthermore, measurement results which were captured during the experiments are used to calibrate various influencing parameters in the simulation.

Fig. 1 shows the schematic clamping situation of the experiments. The two sheets that are to be joined are indicated in the figure. Additionally a third sheet - marked as supporting sheet in the figure - can be seen in the back. These three sheets are mounted onto the support surface by two clamps. The supporting sheet is used to allow an easier experimental performance. In this sheet the pin of the tool plunges in vertically at the beginning of the friction stir welding operation. After that the tool moves in a horizontal motion along the joining line of the two sheets. By the use of a supporting sheet a stable welding process can be achieved in the experiments. The supporting sheet can either be considered or omitted in the simulation. To clarify this aspect, both variations will be examined in the course of the project. For the investigations in this paper the latter case was selected.

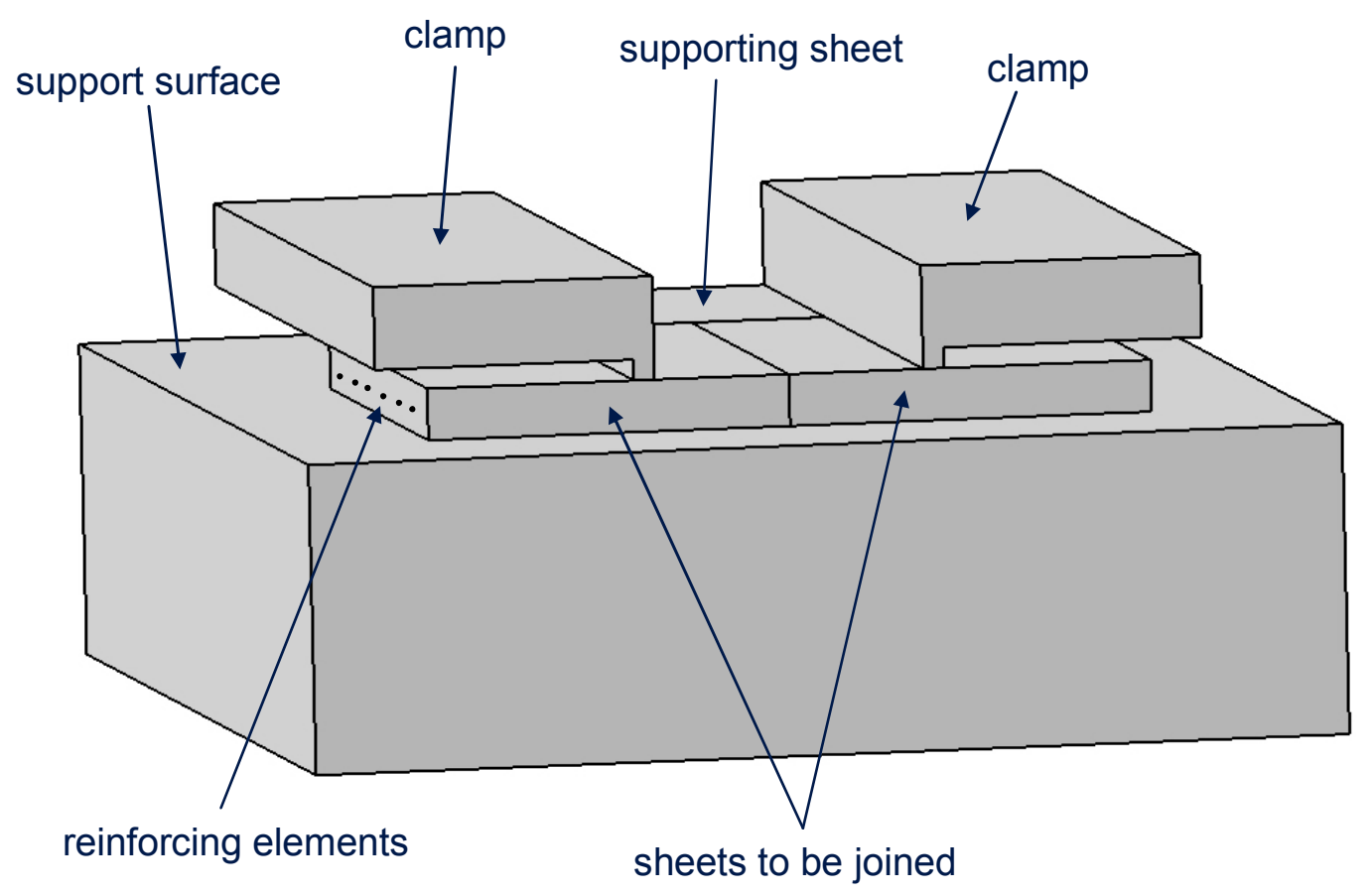

Fig. 1: Schematic clamping situation for the regarded friction stir welding experiments

The definition of the clamping situation plays an important roll in the simulation. The sheets are fixed tightly so that no movement during the welding process is allowed. Through this setup the heat transfer is specified. There are three different ways of heat transfer [2]. The majority of the heat that is input into the structure by the welding process will be transferred from the sheets to the support surface through heat conduction. The contact area between each clamp and the sheets is a thin rectangle that is smaller than the area between the sheets and the support surface. Nevertheless heat conduction will occur through these two areas as well. In addition to conduction, heat is also transferred from the sheets to the surroundings via convection and radiation, although its contribution to the overall heat transfer is lower - taking into account the temperature range that is 
attained during the welding process up to a maximum of $500^{\circ} \mathrm{C}$ for aluminium alloys - than that of heat conduction. Nevertheless, once the workpiece is unclamped, heat transfer will occur only through convection and radiation.

All experimental process parameters that are of importance for the simulation are going to be adopted for the modelling in this case. These parameters are listed in Table 1 below.

\begin{tabular}{|l|r|}
\hline \multicolumn{1}{|c|}{ parameter } & \multicolumn{1}{|c|}{ value [unit] } \\
\hline feed of the tool & $200[\mathrm{~mm} / \mathrm{min}] \approx 3.33[\mathrm{~mm} / \mathrm{s}]$ \\
\hline length of the weld seam & $56[\mathrm{~mm}]$ \\
\hline sheet thickness & $5[\mathrm{~mm}]$ \\
\hline reinforcing element diameter & $1[\mathrm{~mm}]$ \\
\hline initial temperature & $20\left[{ }^{\circ} \mathrm{C}\right]$ \\
\hline
\end{tabular}

Table 1: Selected process parameters of the friction stir welding experiments

\section{Modelling of the friction stir welding}

The first step in the modelling is the generation of an appropriate mesh of solid elements for the composite structure. Usually the mesh is subdivided into two sections - a fine mesh and a coarse mesh. The fine mesh is used to handle the high temperature gradients that appear in the elements where the heat source is applied. Once such a mesh for a single sheet is generated, the mesh of the second sheet is created by mirroring the whole mesh structure in respect to the weld line. If a weld gap shall be considered, solid elements need to be implemented between the two sheets. The handling of the material behaviour of these elements is described below. In addition to the solid elements, groups of two-dimensional elements are defined in order to model the heat exchange. Finally, to describe the welding trajectory, groups of one-dimensional elements are defined. Two nodes indicate where the trajectory starts and where it ends respectively. In the intended simulation the trajectory is a straight line.

Three different materials have to be specified for the simulation. The aluminium matrix consists of EN AW-6060 and the reinforcing steel elements are made of S $355 \mathrm{~J} 2$. If a weld gap should be considered, the elements defined between the two sheets need to be handled by setting Young's modulus to a value that corresponds to $0.7 \%$ of Young's modulus of aluminium [3]. To define the material properties, appropriate databases are used. These are included in the commercial software, which was referred to above, and are complemented by experimental data provided by sub-project A3.

Furthermore, the heat input needs to be defined. As in the first phase of sub-project B4, a conical heat source is one type of heat source that can be used [4]. Fig. 2 illustrates such a heat source and indicates the parameters that define it. These parameters are the maximum source intensity $Q_{0}$ $\left[\mathrm{W} / \mathrm{mm}^{3}\right]$ and four geometrical parameters that define the position relative to the workpiece and the shape of the cone. 


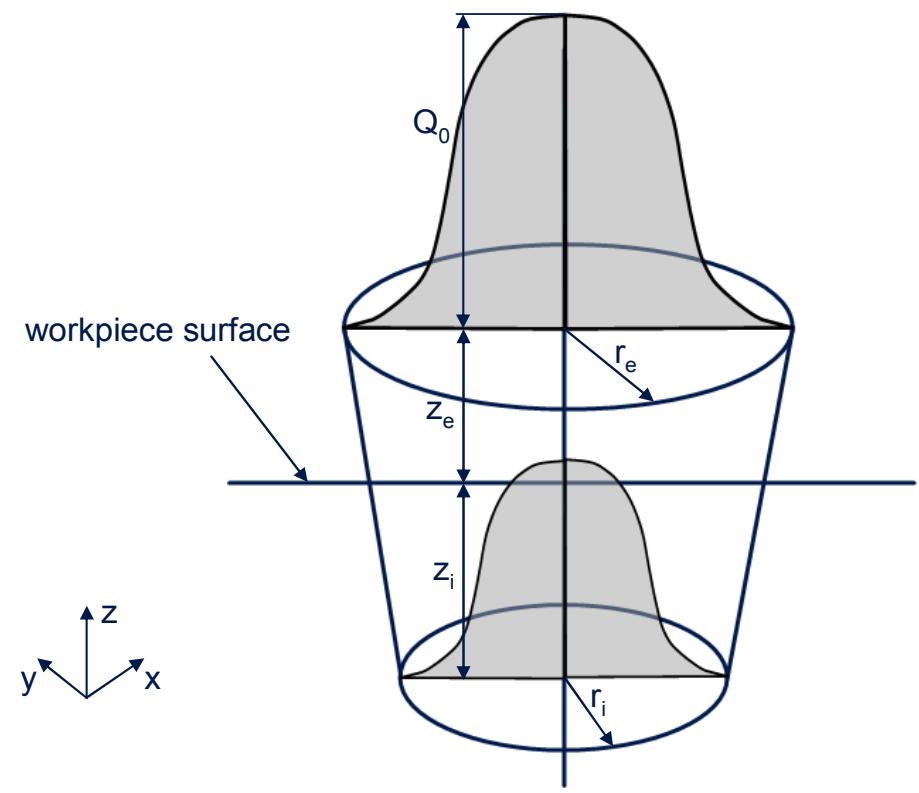

Fig. 2: Conical heat source with its defining parameters

The five defining parameters are determined using cross sections which are provided by subproject A11 and temperature measurements that were generated by various friction stir welding experiments at the authors' institute [5]. In addition to the conical heat source other types of heat sources are going to be tested in order to replicate the real process as close as possible. The influence of a cross section for the parameter definition is discussed in the following. Such a cross section is shown in Fig. 3.

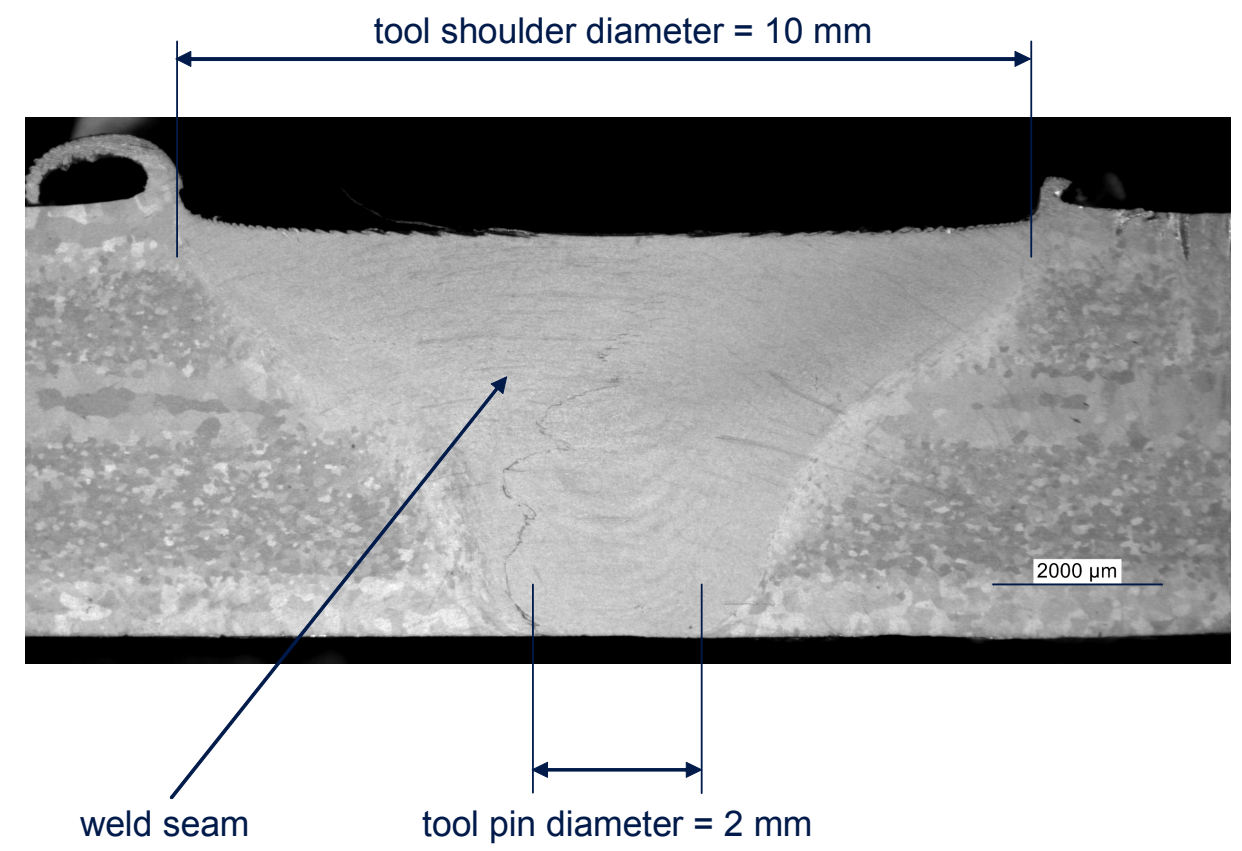

Fig. 3: Cross section of a weld seam from a friction stir welding experiment 
The shape of the weld seam that can be observed in Fig. 3 is typical for friction stir welding. Both the tool shoulder diameter and the tool pin diameter are indicated in the figure. These diameters are mainly responsible for the shape of the weld seam. For a good recreation of the real welding process the parameters of the used heat source have to be chosen in a way that the shape of the temperature field, which is calculated during the simulation, matches well with the shape of the weld seam in the cross section. More heat sources of varying types can be combined simultaneously to accomplish this intention.

\section{Outlook}

Both friction stir welding and laser beam welding are complex processes each with its own specific effects that need to be taken into consideration within a simulation. As an example for such a specific effect the rotation of the friction stir welding tool was examined. As the rotating tool moves along the workpiece it causes a material flow in the welding zone. This process phenomenon is very complex to model and, therefore, it is difficult to be included in a simulation. As usual, various simulations have to be conducted to quantify the different influences and to decide which effects are important and should be considered in the simulation and which are of minor or no importance and can therefore be neglected. In addition to that, some new aspects have to be taken into account in future investigations. The fact that the composite extruded profiles are made of different materials with different material properties leads to additional structural effects that have to be taken into consideration. Taking into account these influencing factors will yield accurate results of the examined welding processes.

\section{Acknowledgement}

This paper is based on investigations of the Transregional Collaborative Research Centre 10 which is kindly supported by the German Research Foundation (DFG).

\section{References}

[1] S. Roeren: Komplexitätsvariable Einflussgrößen für die bauteilbezogene Struktursimulation thermischer Fertigungsprozesse. Diss. Technische Universität München. Herbert Utz Verlag, München, 2007, ISBN 978-3-8316-0680-1, iwb Forschungsbericht Nr. 203.

[2] D. Radaj: Heat Effects of Welding: Temperature Field, Residual Stress, Distortion. SpringerVerlag Berlin Heidelberg 1992, ISBN 3-540-54820-3.

[3] L. Papadakis, S. Roeren: Anwendungsnahe Modelle zur FEM-Berechnung des Verhaltens gefügter Bauteile beim Laserstrahlschweißen. Tagungsband SYSWELD Forum 2005, S. 37-46, Weimar, $1^{\text {st }}$ September 2005.

[4] SYSWELD Reference Manual. ESI Group, 2000.

[5] P. Gebhard, M. F. Zaeh: Empirical Model for the Tool Shoulder Temperature during Friction Stir Welding. Sixth International Symposium on Friction Stir Welding 2006, Saint-Sauveur, $10^{\text {th }}-13^{\text {th }}$ October 2006. 